\title{
Transfer doping of a metallic carbon nanotube and graphene on metal surfaces
}

\author{
Masayuki Hasegawa ${ }^{1, *}$ and Kazume Nishidate ${ }^{2}$ \\ ${ }^{1}$ Faculty of Engineering, Iwate University, Morioka 020-8551, Japan \\ ${ }^{2}$ Department of Electrical Engineering and Information Science, Iwate University, Morioka 020-8551, Japan
}

(Received 14 January 2011; revised manuscript received 3 March 2011; published 20 April 2011)

\begin{abstract}
In this paper we show the results of systematic investigations for the electronic-structure modifications of an armchair $(10,10)$ single-walled carbon nanotube (SWNT) and graphene adsorbed on metal surfaces. The first-principles calculations based on the density-functional theory (DFT) were used to investigate transfer doping of these nanomaterials adsorbed on the fcc (111) surfaces of $\mathrm{Al}$, noble metals ( $\mathrm{Ag}, \mathrm{Cu}$, and $\mathrm{Au}$ ), and transition metals (Rh, Pd, Ir, and Pt). We confirmed that the SWNT weakly interacts with the surfaces of $\mathrm{Al}$ and the noble metals (physisorption), while it strongly bonds to the transition-metal surfaces (chemisorption). The graphene adsorption on these metal surfaces is reinvestigated and found to be similar except for the Ir and Pt substrates, for which the interaction is weak as in the case of $\mathrm{Al}$ and the noble metal substrates. A phenomenological model is also developed on the basis of the rigid-band picture appropriate to physisorption. This model provides a relation between the Fermi-level shift and work-function difference and can conveniently be used in interpreting the DFT results for the transfer doping of both a SWNT and graphene on metal surfaces. We also identify the effect of hybridization between the graphene $\pi$ orbitals and metallic valence states on the Fermi-level shift and find that the hybridization induces an extra downward shift of the linear-dispersion bands near the Dirac point relative to the other bands.
\end{abstract}

DOI: 10.1103/PhysRevB.83.155435

PACS number(s): 73.22.-f, 73.22.Pr, 73.20.At, 73.30.+y

\section{INTRODUCTION}

Since their discovery and successful isolation, carbon nanotubes $(\mathrm{CNTs})^{1}$ and graphene, ${ }^{2}$ a single layer of graphite, have attracted enormous attention because of their unique properties $^{3-5}$ and potential applications in nanoelectronics and other fields of nanotechnology. ${ }^{5-7}$ CNTs are either metallic or semiconducting depending on the chirality, ${ }^{3}$ while graphene is a zero-gap semiconductor with a vanishing density of states at the Femi level. ${ }^{5}$ The electronic properties of these pristine nanostructures are easily modified by mechanical strains, deformations, metal contact, adsorption of foreign atoms or molecules, electric field, and other physical contacts with the environment. These phenomena, originating from nanoscale size and low dimensionality of CNTs and graphene, have been the subject of intensive studies, both experimental and theoretical. Of particular interest are the effects of substrate and metal contact, which are unavoidable in most experiments and possible device applications. These easy modifications do not necessarily deteriorate the usefulness of the nanomaterials, but one can make use of these effects to control their electronic properties, thereby extending a range of technological applications. We focus on metal contact and consider the situation where a metallic CNT or graphene is deposited (or epitaxially grown) on metal surfaces. Electronic and morphological properties of these interfaces have been issues of interest and a number of theoretical and experimental investigations have been made for both $\mathrm{CNTs}^{8-21}$ and graphene $\mathrm{e}^{5,22-39}$ on metal surfaces. Earlier investigations for graphene on metals are reviewed in Refs. 5,22, and 23 and some of the more recent ones are given in Refs. 24-39. Electronic structures of metallic armchair CNTs and graphene are similar in that both have energy bands with linear dispersion crossing at a point in the one- and two-dimensional Brillouin zones. ${ }^{3,5}$ The Fermi levels are located at the point of band crossing, which is called the Fermi point (CNTs) or Dirac point (graphene). The density of states (DOS) of armchair CNTs is flat and small in the vicinity of the Fermi point, while the DOS of graphene vanishes at the Dirac point and linearly increases on both higher- and lower-energy sides, indicative of a semimetallic nature. These electronic structures are prone to modifications by external or internal perturbations, even if they are small. One of such modifications for CNTs and graphene on metallic substrates results from charge transfer, whose nature has been discussed primarily on the basis of the work-function (WF) difference between the adsorbate and substrate. ${ }^{12}$ However, the WF difference alone cannot explain the nature of transfer doping and more detailed understanding of the related interfacial properties will be required for the analyses of experiments and future technological applications of CNTs and graphene. ${ }^{21,25}$

In this paper we use first-principles calculations based on the density-functional theory (DFT) to clarify morphological and electronic properties of a metallic single-walled CNT (SWNT) and graphene adsorbed on metal surfaces. We choose an armchair $(10,10)$ SWNT with a typical size $(\sim 14 \AA$ in diameter) and consider the (111) surfaces of fcc metals, including a simple $s p$ metal $(\mathrm{Al})$, noble metals $(\mathrm{Cu}, \mathrm{Ag}$, and $\mathrm{Au})$, and $4 d$ and $5 d$ transition metals ( $\mathrm{Rh}, \mathrm{Pd}, \mathrm{Ir}$, and $\mathrm{Pt}$ ). These metals have most often been used as electrodes and supporting substrates in both experiments and theoretical investigations, ${ }^{8,23}$ and are of practical use in device fabrications. ${ }^{6,7}$ In the present work, $3 d$ transition metals are not considered partly because of their different natures such as magnetic and highly catalytic properties, which will be an issue of separate investigations. It is intended to find a systematic behavior, if any, in transfer doping and morphological properties in the interface of a SWNT or graphene and metal surfaces. To this end we also use a phenomenological model, ${ }^{21,25}$ which has been developed on the basis of a rigid-band picture and provides useful insight into the nature of transfer doping. The DFT calculations are critically compared with the previous calculations and 
experiments, if available, and are used in the analyses in terms of a phenomenological model.

In the next section we present the details of the DFT electronic-structure calculations for the adsorption of a SWNT and graphene on metal surfaces and summarize the phenomenological model. In Sec. III we show the results of DFT calculations and discuss these results by comparing them with the previous investigations and the phenomenological model. The final section is devoted to the concluding remarks.

\section{METHODOLOGY}

\section{A. DFT calculations}

In the DFT electronic-structure calculations, metal substrates with a (111) surface were modeled by a six-layer slab with $A B C$ stacking, on which an armchair $(10,10)$ SWNT and graphene are deposited, and supercells consisting of this slab, an adsorbate, and the vacuum region of $\sim 12 \AA$ or more over the adsorbate were constructed. The surface structure of a supercell used for graphene/ $\mathrm{Cu}(111)$ consists of a graphene unit cell on a $\mathrm{Cu}(111)$ unit cell. For other metal substrates, we used a supercell system of $2 \times 2$ graphene cells accommodated on the $(\sqrt{3} \times \sqrt{3}) R 30^{\circ}$ substrate structure. The use of these supercells is a compromise between minimizing the effect of lattice mismatch and saving computational cost. In the case of an armchair nanotube on a $\mathrm{Cu}(111)$ surface, the tube axis is aligned along one of the lattice vectors. For other metal surfaces, the tube axis is aligned along one of the lattice vectors of a $(\sqrt{3} \times \sqrt{3}) R 30^{\circ}$ structure, which accommodates two unit cells of a nanotube, as illustrated in Fig. 1. In all cases, the lattice constants of graphene and an armchair SWNT were fixed at their experimental values $(2.46 \AA$ for both) and those of metal surfaces were adjusted to achieve a commensurate supercell structure. Then, the effect of lattice

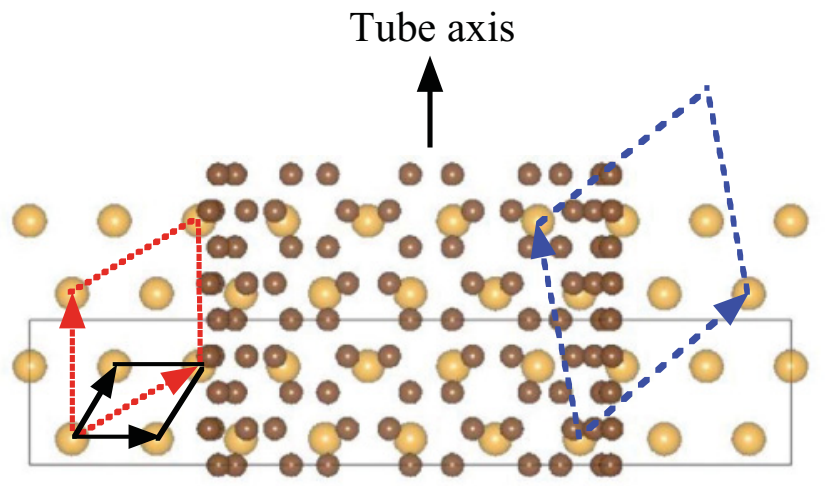

FIG. 1. (Color online) Top view of the metallic (111) surface with an adsorbed $(10,10)$ nanotube: The large (small) balls represent the metal (carbon) atoms and only the carbon atoms of the lower half of the nanotube are shown. The parallelogram with thick solid (black) lines shows a $(1 \times 1)$ unit cell of the $(111)$ surface, and those with dotted (red) and dashed (dark blue) lines show $(\sqrt{3} \times \sqrt{3}) R 30^{\circ}$ and $(\sqrt{7} \times \sqrt{7}) R 19.1^{\circ}$ supercells, respectively, used in the calculations for graphene/metal. The rectangle with thin solid lines represents a supercell used for nanotube/metal calculations, in which the nanotube axis is aligned along one of the lattice vectors of the $(\sqrt{3} \times \sqrt{3}) R 30^{\circ}$ structure. Two supercells are repeated for clarity. mismatch results in $\sim 4 \%$ tensile strain for $\mathrm{Cu}(111), 1-2 \%$ compressive strain for $\mathrm{Al}, \mathrm{Ag}$, and $\mathrm{Au}(111)$, and 2-6\% tensile strain for $\mathrm{Rh}, \mathrm{Pd}, \mathrm{Ir}$, and $\mathrm{Pt}(111)$. To see the effect of lattice mismatch we also used a supercell consiting of $3 \times 3$ graphene unit cells accommodated on the $(\sqrt{7} \times \sqrt{7}) R 19.1^{\circ}$ surface structure (Fig. 1), which results in much reduced tensile strains of $0.5-1.0 \%$ for $\mathrm{Pd}$ and $\mathrm{Pt}(111)$ and $3-4 \%$ for $\mathrm{Rh}$ and $\operatorname{Ir}(111)$. Under these constraints in the lateral plane, geometrical structures of supercells were fully optimized, in which the bottom two layers were fixed. The use of these supercells is a common practice and is nothing but a convention for electronic-structure calculations. In fact, such a periodic structure is not necessarily established in actual situations, and the orientations of nanotubes and graphene on surfaces, either mechanically deposited or epitaxially grown, are not necessarily controllable. In the case of graphene epitaxially grown on metals, lattice mismatch usually leads to a Moire superstructure with long periodicity and often induces out-ofplane buckling (e.g., Refs. 33, 35, and 36). We expect that the results obtained for the prescribed interface configurations are insensitive to the orientation of graphene and are reminiscent of those actually obtained for flat interfaces.

All the DFT [local-density approximation (LDA)] calculations in the present work were performed using the Vienna $a b$ initio simulation package (VASP), ${ }^{40}$ with the exchange-correlation energy functional of Ceperley and Alder ${ }^{41}$ as parametrized by Perdew and Zunger. ${ }^{42}$ We used the projector-augmented wave method ${ }^{43}$ in modeling electron-ion interaction and the Monkhorst-Pack (MP) method ${ }^{44}$ in the k-point sampling in the irreducible Brillouin zone. We used the cutoff energy of $E_{\text {cut }}=300 \mathrm{eV}$, which limits the plane-wave expansion. Varying approximations, depending on the type of calculations, were used in the k-point sampling of the MP method. In the geometrical optimization for nanotube/metals, we used the $\mathbf{k}$ points generated from $1 \times 10 \times 1$ and $1 \times 5 \times 1$ meshes for nanotube/Cu(111) and nanotube/other metals, respectively. In case of graphene/metals, we used $10 \times 10 \times 1,5 \times 5 \times 1$, and $4 \times 4 \times 1 \mathrm{MP}$ meshes for graphene- $(1 \times 1) / \mathrm{Cu}(111), \quad$ graphene- $(2 \times 2) /(\sqrt{3} \times$ $\sqrt{3}) R 30^{\circ}$, and graphene- $(3 \times 3) /(\sqrt{7} \times \sqrt{7}) R 19.1^{\circ}$, respectively. In the electronic-structure calculations we employed more elaborate k-point meshes, which will be mentioned separately. These geometrical optimizations were made within the accuracy that the residual force acting on each atom decreases below $0.01 \mathrm{eV} / \AA$ and dipole corrections appropriate for slab geometry were added in all the DFT calculations.

\section{B. Phenomenological model}

Khomyakov et al. ${ }^{25}$ have developed a phenomenological model for the transfer doping of graphene adsorbed on metal surfaces. The model is based on the rigid-band picture appropriate to physisorption, in which the energy-band structures of an adsorbate and substrate are virtually preserved on contact with each other. In the previous work we have also developed a similar model and successfully applied it to a nanotube and graphene on $\mathrm{Au}(100)$ and $\mathrm{Ag}(100)$ surfaces. $^{21}$ This model is rederived and outlined in the following. We first consider the nanotube adsorption on metal surfaces and, following Khomyakov et al., ${ }^{25}$ start with defining a downward potential 
(a) $p$-type

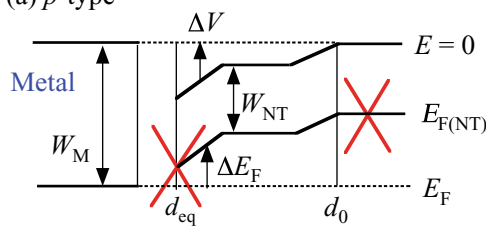

(b) $n$-type

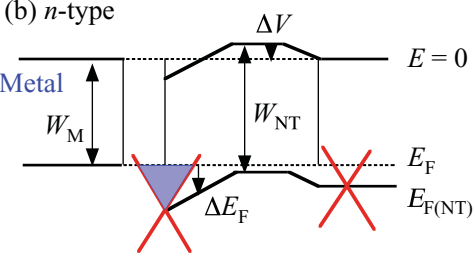

FIG. 2. (Color online) Schematic representation of the Fermilevel alignment and transfer doping for an armchair nanotube (NT) on a metal surface: (a) $p$-type doping and (b) $n$-type doping of the nanotube. As the nanotube is brought closer to a metal surface, the interaction between them comes into force at some distance, $d_{0}$, and the Fermi levels of both systems are aligned at the Fermi level of the combined system [Eq. (2)]. The Fermi-level shift is defined as $\Delta E_{\mathrm{F}}=E_{\mathrm{F}(\mathrm{NT})}-E_{\mathrm{F}}$, where $E_{\mathrm{F}(\mathrm{NT})}$ and $E_{\mathrm{F}} \equiv E_{\mathrm{F}(\mathrm{NT} / \mathrm{M})} \approx E_{\mathrm{F}(\mathrm{M})}$ are the Fermi-point energy of a nanotube and the Fermi energy of the combined system, respectively.

step, $\Delta V$, in the nanotube, i.e., the potential in the nanotube is given by $-\Delta V$ with respect to the substrate. This potential step is interpreted as arising from the interaction between the nanotube and substrate and is assumed to consist of the two contributions:

$$
\Delta V=\Delta V_{\mathrm{c}}+\Delta V_{\mathrm{tr}} .
$$

The first term, $\Delta V_{\mathrm{c}}$, describes a short-ranged repulsion due to the overlap of the nanotube and substrate wave functions and depends primarily on the distance between them. The second term, $\Delta V_{\mathrm{tr}}$, represents a potential step due to dipolelike layered charges induced by charge transfer. The Fermi level should be aligned if the nanotube and substrate begin to communicate with each other at a certain separation. The condition of this Fermi-level alignment can be written as (see Fig. 2)

$$
W_{\mathrm{M}}=W_{\mathrm{NT}}+\Delta V+\Delta E_{\mathrm{F}} .
$$

Here, $W_{\mathrm{M}}$ and $W_{\mathrm{NT}}$ are the work functions (WFs) of metal substrate and nonotube, respectively, and $\Delta E_{\mathrm{F}}$ is the Fermilevel shift of the nanotube defined by $\Delta E_{\mathrm{F}}=\varepsilon_{0}-E_{\mathrm{F}}$, where $\varepsilon_{0} \equiv E_{\mathrm{F}(\mathrm{NT})}$ and $E_{\mathrm{F}}$ are, respectively, the Fermi point energy of a nanotube and the Fermi energy of the combined system. We assumed that the Fermi-level shift of the metal substrate, $\Delta E_{\mathrm{M}}$, is small enough to be negligible. As we see later, small finite values of $\Delta E_{\mathrm{M}}$, typically $\sim 0.01 \mathrm{eV}$, found in the supercell calculations may be interpreted as an artifact due to the finitesize effect.

We now calculate the energy cost, $U$, required for charge transfer to occur as a nanotube is brought to the equilibrium separation, $d_{\mathrm{eq}}$. The first contribution to $U$ is, in case of $p$-type doping, the energy gain due to the hole doping, in which electrons are transferred from the nanotube to the substrate states at the Fermi level [see Fig. 1(a)]. In case of $n$-type doping, the substrate electrons at the Fermi level are transferred to the nanotube with an energy cost [Fig. 1(b)]. The energy involved in these doping processes can be expressed as

$$
U_{\mathrm{dope}}=\int_{\varepsilon_{0}}^{E_{\mathrm{F}}}\left(E-E_{\mathrm{F}}\right) \rho_{\mathrm{NT}}(E) d E,
$$

where $\rho_{\mathrm{NT}}(E)$ is the DOS of a nanotube in the rigid-band model. Here we note that the Fermi point, defined as the point at which band crossing occurs, varies with respect to $E_{\mathrm{F}}$ as the separation $s$ between the nanotube and substrate varies (Fig. 1). Another contribution to $U$ is the energy cost (in case of $p$-type doping) required for the transferred electrons to overcome the potential barrier, which itself is induced by charge transfer. Now let $N_{\text {tr }}(s)$ and $\Delta V_{\text {tr }}(s)$ be the number of transferred electrons and the induced potential barrier at a separation $s$. Both $N_{\mathrm{tr}}(s)$ and $\Delta V_{\mathrm{tr}}(s)$ vanish at sufficiently large separations, say $s>d_{0}$, where the nanotube and substrate no longer interact with each other. The energy required to move the nanotube by a small distance, $\Delta s$, from $s$ to $s+\Delta s$ is given by

$$
\frac{d N_{\mathrm{tr}}(s)}{d s} \Delta s \Delta V_{\mathrm{tr}}(s)+N_{\mathrm{tr}}(s) \frac{d \Delta V_{\mathrm{tr}}(s)}{d s} \Delta s .
$$

Here, the first term represents the energy cost for the newly transferred electrons, whose number is given by $\left(d N_{\text {tr }} / d s\right) \Delta s$, to overcome the energy barrier, $\Delta V_{\mathrm{tr}}(s)$. The second term is the energy cost for the electrons already transferred over the barrier $\Delta V_{\mathrm{tr}}(s)$ to overcome the extra barrier, $\left(d \Delta V_{\mathrm{tr}} / d s\right) \Delta s$. Then, the energy cost of bringing the nanotube to the equilibrium separation is calculated as

$$
\begin{aligned}
U_{\mathrm{tr}}\left(d_{\mathrm{eq}}\right) & =\int_{d_{0}}^{d_{\mathrm{eq}}}\left[\frac{d N_{\mathrm{tr}}(s)}{d s} \Delta V_{\mathrm{tr}}(s)+N_{\mathrm{tr}}(s) \frac{d \Delta V_{\mathrm{tr}}(s)}{d s}\right] d s \\
& =\int_{d_{0}}^{d_{\mathrm{eq}}} \frac{d}{d s}\left[N_{\mathrm{tr}}(s) \Delta V_{\mathrm{tr}}(s)\right] d s=N_{\mathrm{tr}}\left(d_{\mathrm{eq}}\right) \Delta V_{\mathrm{tr}}\left(d_{\mathrm{eq}}\right)
\end{aligned}
$$

with the assumption, $N_{\mathrm{tr}}(s)=\Delta V_{\mathrm{tr}}(s)=0$ for $s>d_{0}$. The above result is the same as that previously obtained by a less transparent argument. ${ }^{21}$ Hereafter, $d_{\mathrm{eq}}$ dependence of $U_{\text {tr }}\left(d_{\text {eq }}\right), N_{\text {tr }}\left(d_{\text {eq }}\right)$, and $\Delta V_{\text {tr }}\left(d_{\text {eq }}\right)$ is treated implicitly and these quantities are simply written as $U_{\mathrm{tr}}, N_{\mathrm{tr}}$, and $\Delta V_{\mathrm{tr}}$, respectively. In the rigid-band model, $N_{\text {tr }}$ can be written as

$$
N_{\mathrm{tr}}=-\int_{\varepsilon_{0}}^{E_{\mathrm{F}}} \rho_{\mathrm{NT}}(E) d E .
$$

Both $N_{\text {tr }}$ and $\Delta V_{\text {tr }}$ are, by definition, positive (negative) for the $p$-type ( $n$-type) doping of the nanotube. Before proceeding to the next step, we note that $\Delta V_{\text {tr }}$ is certainly proportional to the number of transferred electrons and may be written as $\Delta V_{\mathrm{tr}}=\alpha N_{\mathrm{tr}}$, where $\alpha$ is a constant depending on the spatial distribution of the transferred electrons and is treated as an unknown parameter. This form of $\Delta V_{\mathrm{tr}}$ and its treatment are crucial in the present phenomenological model. We also note that both $\Delta V_{\mathrm{c}}$ and $\Delta V_{\text {tr }}$ must be interpreted as the averaged quantities since the nanotube contact with a flat surface is not uniform.

The Fermi-level shift, $\Delta E_{F}=\varepsilon_{0}-E_{F}$, or $N_{\text {tr }}$ given by Eq. (5) may be determined by the requirement that the total energy cost, $U=U_{\text {dope }}+U_{\mathrm{tr}}$, is minimum with respect to 
$\Delta E_{\mathrm{F}}$ and $\alpha$ under the constraint of the Fermi-level alignment as given by Eq. (2). We note that both $\Delta E_{\mathrm{F}}$ and $\alpha$ are the parameters related to charge transfer. On the other hand, the work functions (WFs) and $\Delta V_{\mathrm{c}}$ are treated as the given parameters independent of charge transfer itself. We solved the optimization problem using the Lagrange multiplier method and found that $\Delta E_{\mathrm{F}}$ is determined by the equation (the derivation is given in Ref. 21)

$$
\Delta E_{\mathrm{F}}+2 \frac{N_{\mathrm{tr}}\left(\Delta E_{\mathrm{F}}\right)}{\rho_{\mathrm{NT}}\left(E_{\mathrm{F}}\right)}=\Delta W-\Delta V_{\mathrm{c}},
$$

where $\Delta W$ is the WF difference, $\Delta W=W_{\mathrm{M}}-W_{\mathrm{NT}}$. If the Fermi energy $E_{\mathrm{F}}$ is in the range of the linear-dispersion bands of an armchair nanotube, where $\rho_{\mathrm{NT}}(E)$ is nearly flat, we have $N_{\mathrm{tr}}\left(\Delta E_{F}\right) \approx \rho_{N T}\left(E_{F}\right) \Delta E_{F}$. Using this in Eq. (6), we find

$$
\Delta E_{\mathrm{F}} \approx \frac{1}{3}\left(\Delta W-\Delta V_{\mathrm{c}}\right) .
$$

By comparing Eq. (6) with the condition of the Fermi-level alignment [Eq. (2)], we find that

$$
\Delta V_{\mathrm{tr}}=2 \frac{N_{\mathrm{tr}}\left(\Delta E_{\mathrm{F}}\right)}{\rho_{\mathrm{NT}}\left(E_{\mathrm{F}}\right)},
$$

or $\alpha=2 / \rho_{\mathrm{NT}}\left(E_{\mathrm{F}}\right)$, indicating that $\Delta V_{\text {tr }}$ is twice as large as the Fermi-level shift itself for an armchair nanotube with a flat DOS in the neighborhood of the Fermi point.

In the case of the graphene adsorption on metal surfaces, the condition of the Fermi-level alignment is written as $W_{\mathrm{M}}=W+\Delta V$, in place of Eq. (2), where $W$ is the work function of the metal surface with adsorbed graphene and $\Delta V$, defined by this relation, represents the potential step across the substrate-graphene interface. Therefore, $\Delta V$ corresponds to $\Delta V_{\mathrm{NT}}$ and $W$ to $W_{\mathrm{G}}+\Delta E_{\mathrm{F}}$, and the phenomenological model for the nanotube adsorption can straightforwardly be applicable to the graphene adsorption by simply replacing $W_{\mathrm{NT}}$ by $W_{\mathrm{G}}$ and $\rho_{\mathrm{NT}}(E)$ by $\rho_{\mathrm{G}}(E)$, where $W_{\mathrm{G}}$ and $\rho_{\mathrm{G}}(E)$ are the graphene WF and DOS, respectively. The present phenomenological model is similar to, but somewhat different from, that used by Khomyakov et al. ${ }^{25}$ in their analyses of the graphene physisorption on metal surfaces. The potential steps, $\Delta V_{\mathrm{c}}$ and $\Delta V_{\mathrm{tr}}$, have been explicitly estimated in their model, whereas we used a variational method to determine $\Delta V_{\mathrm{tr}}$ and $\Delta E_{\mathrm{F}}$, and treated $\Delta V_{\mathrm{c}}$ as the parameter to be determined, if necessary, by other methods. The present model may be better applicable to graphene on metals, in which the potential steps are better defined because of a flat interface. Since the graphene DOS near the conical (Dirac) point shows a linear dependence on the energy and is given by $\rho_{\mathrm{G}}(E) \approx D_{0}\left|E-\varepsilon_{0}\right|$, where $\varepsilon_{0}=E_{\mathrm{F}(\mathrm{G})}$ is now the energy of the conical (Dirac) point, we find $N_{\mathrm{tr}}\left(\Delta E_{\mathrm{F}}\right)=(1 / 2) \rho_{\mathrm{G}}\left(E_{\mathrm{F}}\right) \Delta E_{\mathrm{F}}$ with $\rho_{\mathrm{G}}\left(E_{\mathrm{F}}\right)=$ $D_{0}\left|\Delta E_{\mathrm{F}}\right|$. Using these relations in Eq. (6) with $\rho_{\mathrm{NT}}$ replaced by $\rho_{\mathrm{G}}$, we have

$$
\Delta E_{\mathrm{F}} \approx \frac{1}{2}\left(\Delta W-\Delta V_{c}\right),
$$

where the WF difference is now given by $\Delta W=W_{\mathrm{M}}-W_{\mathrm{G}}$. The above result can be obtained by simply replacing the factor $1 / 3$ in Eq. (7) by 1/2, reflecting flat and linear behaviors of the DOS of an armchair nanotube and graphene. Also note that
$\Delta V_{\mathrm{tr}}=\Delta E_{\mathrm{F}}$ for the graphene adsorption in our model, which is compared to the numerical results, $\Delta V_{\mathrm{tr}} \sim(2 / 3) \Delta E_{\mathrm{F}}$, in the model of Khomyakov et al. ${ }^{25}$

\section{RESULTS AND DISCUSSION}

\section{A. SWNT on metal surfaces}

We chose an armchair $(10,10)$ SWNT of typical size adsorbed on the fcc(111) surfaces of Al, noble metals (Ag, $\mathrm{Cu}$, and $\mathrm{Au}$ ), and $4 d$ and $5 d$ transition metals (Rh, Pd, Ir, and $\mathrm{Pt}$ ). These metal substrates have often been used in experiments and could be useful in some device applications. The electronic-structure calculations for these systems were performed using $1 \times 24 \times 1$ and $1 \times 12 \times 1 \mathbf{k}$-point meshes for nanotube/ $\mathrm{Cu}(111)$ and nanotube on other metal surfaces with $(\sqrt{3} \times \sqrt{3}) R 30^{\circ}$ structure, respectively. The adsorption parameters obtained by these calculations are summarized in Table I. The adsorptions of a nanotube on $\mathrm{Al}$ and noble metal substrates are typical of physisorption with large equilibrium separations, $d_{\mathrm{eq}} \sim 3.0 \AA$, and relatively small adsorption energies. On the other hand, we find that $d_{\mathrm{eq}} \sim 2.0 \AA$ and the adsorption energies are much larger in the adsorptions on transition-metal substrates. These adsorption characteristics of a metallic $(10,10)$ SWNT on $\mathrm{Al}, \mathrm{Au}, \mathrm{Pd}$, and Pt(111) surfaces are similar to those for semiconducting zigzag SWNTs, ${ }^{16-18}$ indicating that these interfacial properties are insensitive to the type and size of SWNTs. Figure 3 illustrates geometrical structures of a nanotube on metal surfaces, showing that the nanotube cross-sectional shape remains circular, although we find small deformations, if any, on the transition-metal surfaces. These results for a $(10,10)$ SWNT on transition-metal surfaces are in contrast to the DFT calculations of Maite and Ricca, ${ }^{16}$ who have demonstrated that a much thinner $(8,0)$ SWNT ( $\sim 6.3 \AA$ in diameter) shows substantial deformations on the $\operatorname{Pd}(111)$ and $\operatorname{Pt}(111)$ surfaces. These deformations may be interpreted as arising from the enhanced $\pi$-orbital distribution outside nanotube. The amplitudes of $\pi$ orbitals are higher outside the nanotube than inside because of the $\pi-\pi$ repulsion (curvature effect) and are further enhanced outside as the nanotube is adsorbed on a substrate and $\pi$ orbitals strongly hybridize with the substrate wave functions to form bonds to surface atoms. The reduced $\pi-\pi$ repulsion due to the reduced $\pi$-orbital amplitude inside nanotube may allow neighboring atoms to come closer to each other, thereby deforming the nanotube. The curvature effect may not be large enough for a large nanotube to deform even on the transition-metal surfaces, which is probably the case of a $(10,10)$ SWNT on metals. In the case of physisorption, in which no bonding formation occurs, even a nanotube of small diameter is not likely to deform, as demonstrated by the present and previous investigations. ${ }^{16-18}$ The curvature effect on the $\pi$ orbital distribution is also the cause of semiconductor-to-metal transition induced by radial deformation of semiconducting SWNTs. ${ }^{45,46}$ In this case, the lowering of a conduction band due to the enhanced $\pi$-orbital amplitude outside the nanotube, which is more enhanced through a higher local curvature due to increasing radial deformation, is responsible for the transition. 
TABLE I. Summary of the adsorption parameters for an armchair $(10,10)$ nanotube (NT) and graphene on fcc-metal (111) surfaces. $d_{\mathrm{eq}}$ is the calculated equilibrium separation between the nanotube or graphene atoms and the topmost surface layer, $\Delta E_{\text {ad }}$ is the adsorption energy [per NT unit cell for $(10,10) \mathrm{NT} /$ metal and per carbon atom for graphene/metal], and $\Delta E_{\mathrm{F}}$ is the Fermi-level shift. $\Delta W$ is the work-function (WF) difference, $\Delta W=W_{\mathrm{M}}-W_{\mathrm{NT}}$, used in the phenomenological analyses, where $W_{\mathrm{NT}}=4.48 \mathrm{eV}$ and $W_{\mathrm{M}}$ is the WF of the metal (111) surface. The same $\Delta W$ was used for graphene/metal since the graphene $\mathrm{WF}, W_{\mathrm{G}}$, is almost the same as $W_{\mathrm{NT}}$.

\begin{tabular}{|c|c|c|c|c|c|c|c|c|}
\hline Metal & $\mathrm{Al}$ & $\mathrm{Ag}$ & $\mathrm{Cu}$ & $\mathrm{Au}$ & $\mathrm{Ir}$ & $\mathrm{Pt}$ & $\mathrm{Rh}$ & $\mathrm{Pd}$ \\
\hline \multicolumn{9}{|l|}{$(10,10) \mathrm{NT} / \mathrm{metal}$} \\
\hline$d_{\mathrm{eq}}(\AA)$ & 3.21 & 2.91 & 3.05 & 3.04 & 2.01 & 1.98 & 2.00 & 2.05 \\
\hline$\Delta E_{\mathrm{ad}}(\mathrm{eV})$ & 0.23 & 0.26 & 0.58 & 0.25 & 1.68 & 1.15 & 1.98 & 2.57 \\
\hline$\Delta E_{\mathrm{F}}(\mathrm{eV})$ & -0.15 & -0.07 & 0.09 & 0.14 & & & & \\
\hline$\Delta W(\mathrm{eV})$ & -0.22 & 0.26 & 0.46 & 0.83 & 0.42 & 1.60 & 0.92 & 1.12 \\
\hline \multicolumn{9}{|l|}{ Graphene/metal } \\
\hline$d_{\mathrm{eq}}(\AA)$ & 3.41 & 3.33 & 3.26 & 3.31 & 3.23 & 3.21 & $2.13-2.38$ & $2.27-2.49$ \\
\hline$\Delta E_{\mathrm{ad}}(\mathrm{eV})$ & 0.036 & 0.021 & 0.033 & 0.025 & 0.026 & 0.038 & 0.124 & 0.079 \\
\hline$\Delta E_{\mathrm{F}}(\mathrm{eV})$ & -0.52 & -0.30 & -0.17 & 0.20 & 0.05 & 0.29 & & \\
\hline$\Delta E_{\mathrm{F}}(\mathrm{eV})^{\mathrm{a}}$ & -0.57 & -0.32 & -0.17 & 0.19 & & 0.33 & & \\
\hline$\Delta E_{\mathrm{F}}(\mathrm{eV})(\mathrm{expt})$ & & & & $0.35-0.40^{\mathrm{b}}$ & $0.10 \pm 0.02^{\mathrm{c}}$ & $0.30 \pm 0.15^{\mathrm{d}}$ & & \\
\hline
\end{tabular}

\section{${ }^{\mathrm{a}}$ Reference 25 .}

${ }^{\mathrm{b}}$ Reference 34.

${ }^{\mathrm{c}}$ Reference 39 .

${ }^{\mathrm{d}}$ Reference 31 .
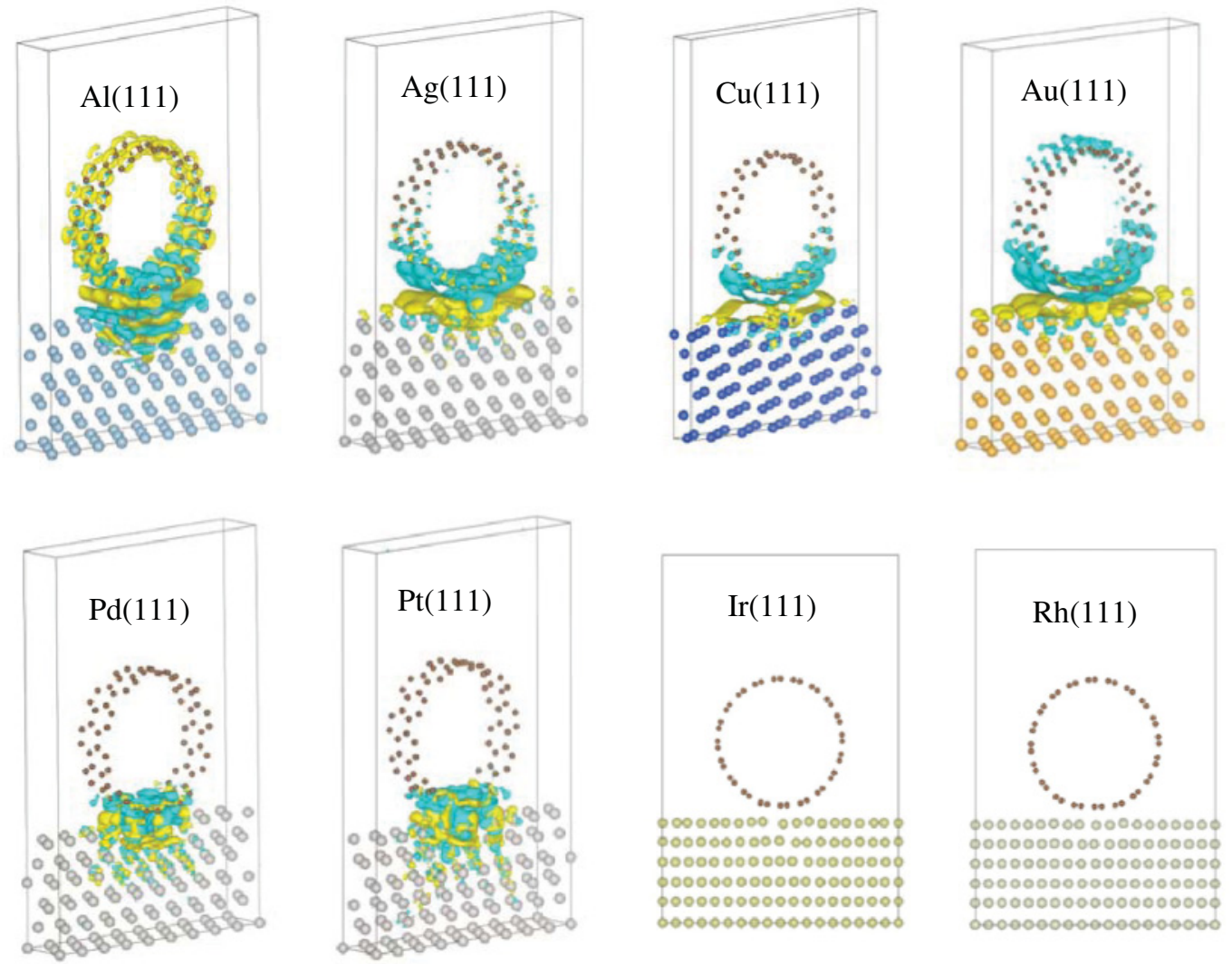

FIG. 3. (Color online) Difference charge densities $\Delta n(\mathbf{r})$, defined by Eq. (10), for the system of a $(10,10)$ armchair nanotube (NT) on metal surfaces. The blue (shaded darker) and yellow (shaded lighter) domains show the electron depleted $[\Delta n(\mathbf{r})<0]$ and electron accumulated $[\Delta n(\mathbf{r})>0$ ] regions, respectively. Only the atomic configurations are shown for NT/Ir(111) and NT/Rh(111), in which $\Delta n(\mathbf{r})$ 's are similar to those for NT/Pd(111) and NT/Pt(111). 
Following the common practice, ${ }^{18}$ we can visualize charge redistribution in terms of the different electron density defined by

$$
\Delta n(\mathrm{r})=n_{\mathrm{NT} / \mathrm{M}}(\boldsymbol{r})-n_{\mathrm{NT}}(r)-n_{\mathrm{M}}(\boldsymbol{r}),
$$

where $n_{\mathrm{NT} / \mathrm{M}}(\mathbf{r})$ is the electron number density of the combined system consisting of the nanotube (NT) and metallic substrate $(\mathrm{M})$, and $n_{\mathrm{NT}}(\mathbf{r})\left[n_{\mathrm{M}}(\mathbf{r})\right]$ is that of the nanotube (substrate) obtained by simply removing the substrate (nanotube) from the atomic configuration of the combined system. Figure 3 illustrates the prominent regions of $\Delta n(\mathbf{r})<0$ and $\Delta n(\mathbf{r})>0$ for the systems under consideration. In the nanotube adsorption on the $\mathrm{Al}$ surface, $\mathrm{NT} / \mathrm{Al}(111)$, electrons are transferred from the substrate to the nanotube and the transferred electrons distribute all over the nanotube. In contrast to this $n$-type doping of the nanotube, $p$-type doping occurs for $\mathrm{NT} / \mathrm{Au}(111)$, where the electrons transferred from the nanotube predominantly distribute in the interface region. The cases of NT/ $\mathrm{Ag}(111)$ and $\mathrm{NT} / \mathrm{Cu}(111)$ are somewhere between NT/Al(111) and NT/Au(111). On the other hand, bonding formation rather than charge transfer occurs for the nanotube on the transition-metal surfaces as exemplified for $\mathrm{NT} / \mathrm{Pd}(111)$ and $\mathrm{NT} / \mathrm{Pt}(111)$ in Fig. 3. In these cases, both domains with $\Delta n(\mathbf{r})<0$ and $\Delta n(\mathbf{r})>0$ are localized in the interface region and bonding formation is clearly seen as the domain with $\Delta n(\mathbf{r})>0$ connecting the nanotube and substrate.

As we have already seen in Table I and Fig. 3, the nanotube adsorptions on the $\mathrm{Al}$ and noble metal surfaces are typical of physisorption with small adsorption energies and rather large contact distance, $d_{\mathrm{eq}} \sim 3 \AA$. In such cases, the electronic structure of the adsorbed nanotube may be more or less preserved as actually suggested by scanning tunneling spectroscopy (STS) measurements on a similar system. ${ }^{15}$ This rigid-band nature can be tested in terms of the DOSs. Figure 4 illustrates the DOS of a nanotube, metallic substrate, and combined system of the nanotube on substrate, which we denote $\rho_{\mathrm{NT}}(E), \rho_{\mathrm{M}}(E)$, and $\rho_{\mathrm{NT} / \mathrm{M}}(E)$, respectively. These DOS correspond to the charge densities defined in Eq. (10) and were obtained by the same calculations. No singular behavior is seen in these DOS because the Gaussian smearing of $0.1 \mathrm{eV}$ width was used in the electronic-structure calculations. We note that each DOS in the left panel of Fig. 4 is shown as a function of $E-E_{\mathrm{F}(i)}$, where $E_{\mathrm{F}(i)}$ is the Fermi energy of each system, i.e., $i=\mathrm{NT}$ (freestanding nanotube), M (isolated metal substrate), or NT/M (system of nanotube/metal), and we use $E_{\mathrm{F}} \equiv E_{F(\mathrm{NT} / \mathrm{M})}$ hereafter. Highly enhanced $\rho_{\mathrm{NT} / \mathrm{M}}(E)$ and $\rho_{\mathrm{M}}(E)$ below the Fermi level, except for NT/Al(111), are the contributions from the valence $d$ states of the metal substrates. The Fermi point of the nanotube is not in the range of these $d$ states, implying that nanotube $\pi$ orbitals do not strongly hybridize with metal $d$ states and bonding is unlikely to form, as actually seen from the charge distributions (Fig. 3). In such a situation, a rigid-band model may conveniently be used in the analysis of charge transfer. The validity of this model can clearly be seen using a superimposed DOS defined by

$$
\rho_{\mathrm{NT} / \mathrm{M}}^{\text {rigid }}(E)=\rho_{\mathrm{NT}}\left(E-\Delta E_{\mathrm{NT}}\right)+\rho_{\mathrm{M}}\left(E-\Delta E_{\mathrm{M}}\right) .
$$

Here, $\rho_{\mathrm{NT}}(E)$ and $\rho_{\mathrm{M}}(E)$ are appropriately shifted by $\Delta E_{\mathrm{NT}}$ and $\Delta E_{\mathrm{M}}$, respectively, toward higher energies and are superimposed to define $\rho_{\mathrm{NT} / \mathrm{M}}^{\mathrm{rigid}}(E)$. As illustrated in the right panel of Fig. 4, $\rho_{\mathrm{NT} / \mathrm{M}}^{\text {rigid }}(E)$ in the vicinity of the Fermi level can almost completely be fitted to $\rho_{\mathrm{NT} / \mathrm{M}}(E)$ using appropriately chosen $\Delta E_{\mathrm{NT}}$ and $\Delta E_{\mathrm{M}}$, indicating that the rigid-band picture is valid. We note that this fit can be achieved in the whole energy range using the same values of $\Delta E_{\mathrm{NT}}$ and $\Delta E_{\mathrm{M}}$, i.e., the whole bands show a uniform shift. As we see later, this is not the case in the graphene adsorption on metals. The energy shifts, $\Delta E_{\mathrm{NT}}$ and $\Delta E_{\mathrm{M}}$, are nothing but so-called Fermi-level shifts of the nanotube and substrate, respectively, which are defined as $\Delta E_{\mathrm{NT}}=E_{\mathrm{F}(\mathrm{NT})}-E_{\mathrm{F}}$ and $\Delta E_{\mathrm{M}}=E_{\mathrm{F}(\mathrm{M})}-E_{\mathrm{F}}$, with $E_{\mathrm{F}}=E_{\mathrm{F}(\mathrm{NT} / \mathrm{M})}$ as before. We find that $\Delta E_{\mathrm{M}}$ is very small, typically $\sim 0.01 \mathrm{eV}$ or smaller, in all the cases and, as we have already mentioned, finite $\Delta E_{\mathrm{M}}$ can be interpreted as an artifact due to finite-size effect of the substrate, which is modeled with a six-layer slab. The Fermi-level shifts of the nanotube, $\Delta E_{\mathrm{F}}=\Delta E_{\mathrm{NT}}$, are summarized in Table I. The positive (negative) values of $\Delta E_{\mathrm{F}}$ indicate $p$-type (n-type) doping of the nanotube (Fig. 1). The predicted $p$-type doping of a metallic nanotube on a $\mathrm{Au}(111)$ surface is consistent with experiments, ${ }^{9,12,13}$ although the predicted Fermi-level shift, $\Delta E_{\mathrm{F}}=0.14 \mathrm{eV}$, is somewhat smaller than the experimental values, $\Delta E_{\mathrm{F}} \approx$ $0.20-30 \mathrm{eV}$.

Figure 5 shows the DFT calculations for the Fermi-level shifts $\Delta E_{\mathrm{F}}$ of the nanotube as functions of the WF difference, $\Delta W=W_{\mathrm{M}}-W_{\mathrm{NT}}$, and their comparisons with the result of the phenomenological model with $\Delta V_{\mathrm{c}}=0.39 \mathrm{eV}$ in Eq. (7). Here, we used $W_{\mathrm{NT}}=4.48 \mathrm{eV}$ obtained by the present DFT calculations, which is in good agreement with the previous ones obtained by similar calculations. ${ }^{47-49}$ The WF differences used in our analyses are given in Table I. These results were obtained by using the above value of $W_{\mathrm{NT}}$ and experimental values for the metal (111) surfaces. ${ }^{50}$ The DFT results for $\Delta E_{\mathrm{F}}$ are roughly on a single line, indicating that the values of $\Delta V_{\mathrm{c}}$ are similar for all cases under consideration. In more detail, the value of $\Delta V_{\mathrm{c}}$ for an $\mathrm{Al}(111)$ surface are a little smaller than those for noble metal surfaces, suggesting a reduced repulsion due to the extended nature of the $\mathrm{Al} s p$ states. The validity of the phenomenological model can be assessed by using Eq. (6), which implies that the nanotube DOS near the Fermi point, which has a major influence on doping characteristics, plays a crucial role in determining the Fermi-level shift. This can be demonstrated using the results shown in Fig. 6, which are the counterparts of those in Fig. 4 and were obtained by less accurate electronic-structure calculations using a $1 \times 5 \times 1 \mathrm{MP}$ k-point mesh. In these calculations the flat DOS of an armchair SWNT near the Fermi point is not achieved. The resulting Fermi-level shifts are -0.25 and $0.23 \mathrm{eV}$ for NT/Al(111) and $\mathrm{NT} / \mathrm{Au}(111)$, respectively, which are much larger in magnitude than the accurate calculations (Fig. 4). Using these DOS and the resulting $N_{\text {tr }}\left(\Delta E_{\mathrm{F}}\right)$ in Eq. (6), we have $\Delta W-$ $\Delta V_{\mathrm{c}}=-0.42$ and $0.45 \mathrm{eV}$ for NT/Al(111) and NT/Au(111), respectively. We now assume that the quantity, $\Delta W-\Delta V_{\mathrm{c}}$, which is implicit in the electronic-structure calculations, is not so sensitive to the approximation involved in the DFT 

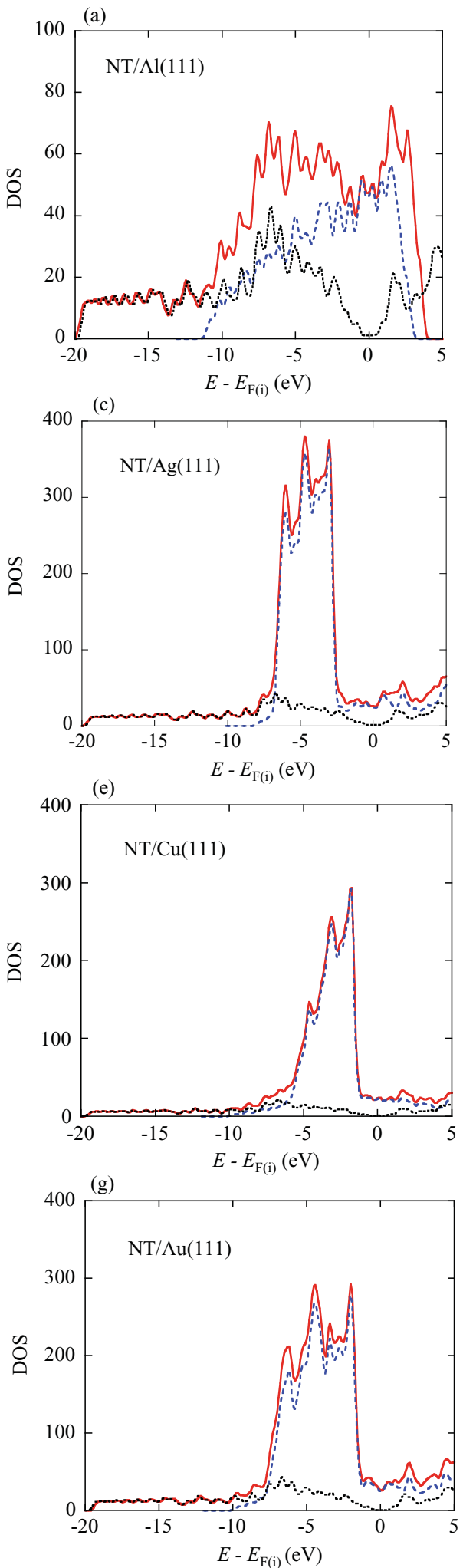
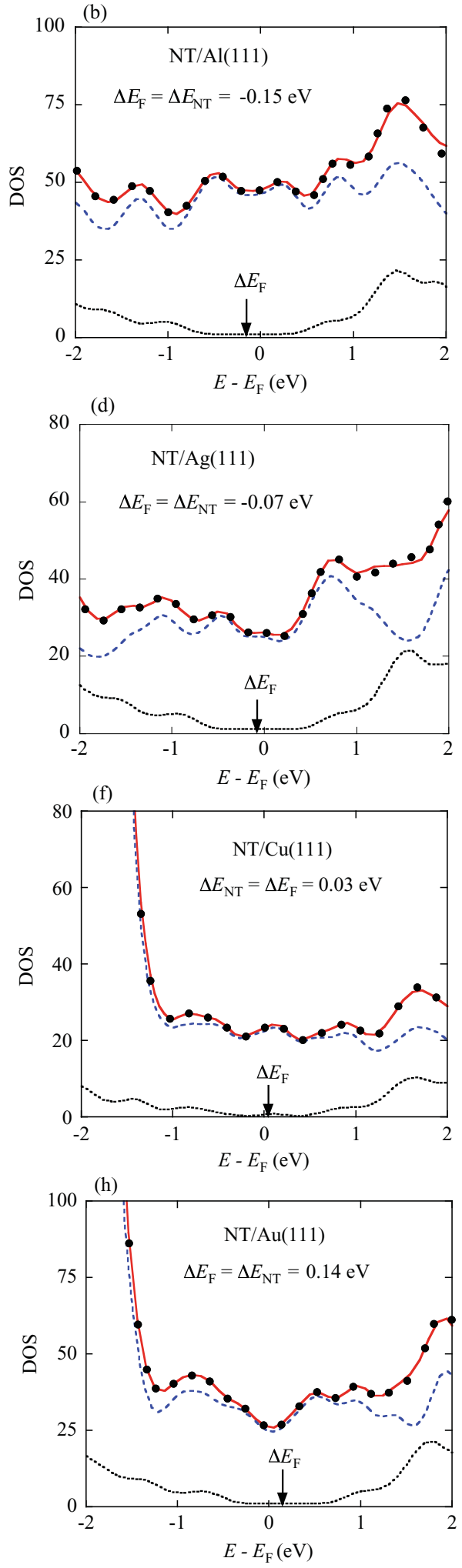

FIG. 4. (Color online) Density of states (DOS) of the systems of an armchair $(10,10)$ nanotube $(\mathrm{NT})$ on $\mathrm{Al}, \mathrm{Ag}$, $\mathrm{Cu}$, and $\mathrm{Au}(111)$ surfaces. Red (solid), blue (dashed), and black (dotted) lines show the DOS of the combined system $\left[\rho_{\mathrm{NT} / \mathrm{M}}(E)\right]$, metallic substrate $\left[\rho_{\mathrm{M}}(E)\right]$, and nanotube $\left[\rho_{\mathrm{NT}}(E)\right]$, respectively. The atomic configurations of the latter two cases are those obtained by removing, respectively, nanotube and substrate from the combined system. In the left panel, $E_{\mathrm{F}(i)}$ is the Fermi level of each system: $i=\mathrm{NT} / \mathrm{M}$ (combined system), NT (nanotube only), or M (metallic substrate only). In the right panel, $E_{\mathrm{F}} \equiv E_{\mathrm{F}(\mathrm{NT} / \mathrm{M})}$ is the Fermi level of the combined system, and $\rho_{\mathrm{NT}}(E)$ and $\rho_{\mathrm{M}}(E)$ are appropriately shifted by $\Delta E_{\mathrm{NT}}$ and $\Delta E_{\mathrm{M}}$, respectively, so that the superimposed DOS, $\rho_{\mathrm{NT} / \mathrm{M}}^{\text {rigid }}(E)$, defined by Eq. (10) is close to $\rho_{\mathrm{NT} / \mathrm{M}}(E)$ near the Fermi level. Solid circles show $\rho_{\mathrm{NT} / \mathrm{M}}^{\text {rigid }}(E)$ obtained in this way and are in good accordance with $\rho_{\mathrm{NT} / \mathrm{M}}(E)$ in the whole energy range, indicating the validity of rigid band model. Vertical arrows show the Fermi-level shift, $\Delta E_{\mathrm{F}}$. calculations. Then, using the above values of $\Delta W-\Delta V_{\mathrm{c}}$ in Eq. (7) appropriate to flat (accurate) DOS, we have $\Delta E_{\mathrm{F}}=-0.14$ and $0.15 \mathrm{eV}$ for $\mathrm{NT} / \mathrm{Al}(111)$ and $\mathrm{NT} / \mathrm{Au}(111)$, respectively, which are in good agreement with the accurate calculations (Fig. 4). These analyses demonstrate the validity as well as usefulness of the present phenomenological model.

\section{B. Graphene on metals}

Interfacial characteristics of graphene on metals were reexamined by essentially the same type of DFT calculations as the previous ones, ${ }^{24,25}$ but we made somewhat different analyses in terms of DOS and the phenomenological model. More accurate calculations are also 


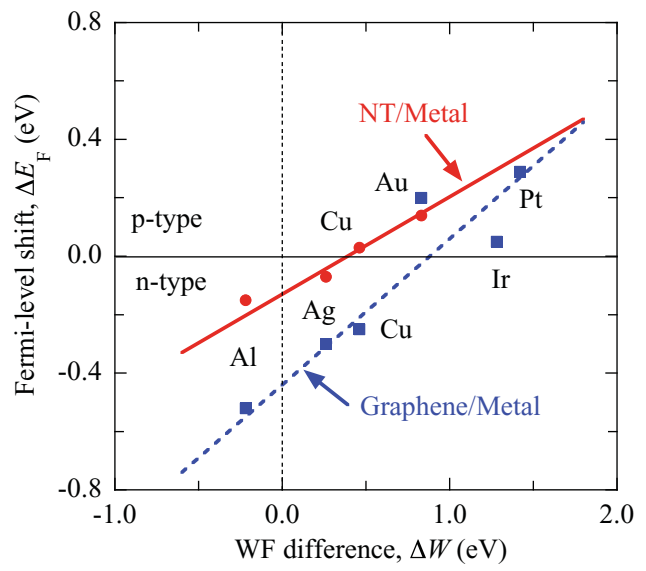

FIG. 5. (Color online) Fermi-level shifts vs work-function (WF) difference for the systems of nanotube (NT)/metals and graphene/metals. Solid circles and squares show the results of DFT calculations for $\mathrm{NT} / \mathrm{metals}$ and graphene/metals, respectively. The red (solid) and dark blue (dashed) lines represent, respectively, the results given by Eq. (7) with $\Delta V_{\mathrm{c}}=$ $0.39 \mathrm{eV}$ and Eq. (9) with $\Delta V_{\mathrm{c}}=0.88 \mathrm{eV}$.

attempted using a larger supercell with smaller effect of lattice mismatch. In the DFT electronic-structure calculations, we used $36 \times 36 \times 1,24 \times 24 \times 1$, and $16 \times 16 \times 1 \mathrm{MP} \mathbf{k}$-point meshes, which correspond to graphene $/ \mathrm{Cu}(111)$, graphene$(2 \times 2) /(\sqrt{3} \times \sqrt{3}) R 30^{\circ}$ for the $\mathrm{Al}$ and noble metal surfaces, and graphene- $(3 \times 3) /(\sqrt{7} \times \sqrt{7}) R 19.1^{\circ}$ for the transitionmetal surfaces, respectively. The adsorption parameters obtained by these calculations are summarized in Table I. These results are similar to the previous calculations ${ }^{25}$ except for $\mathrm{Rh}(111)$ and $\operatorname{Ir}(111)$ surfaces, which are not available. The equilibrium distances $d_{\mathrm{eq}}$ between the adsorbed graphene and substrates are 3.2-3.4 $\AA$ for the $\mathrm{Al}$ and noble metal substrates, indicative of weak interaction as in the case of the nanotube adsorptions. For the graphene adsorptions on $\operatorname{Ir}(111)$ and $\operatorname{Pt}(111)$ surfaces, the equilibrium distances are also large $\left(d_{\mathrm{eq}}=3.1-3.2 \AA\right)$ and the adsorption energies are small, which are typical features of physisorption and are in contrast to the nanotube adsorptions on those metal surfaces. For the $4 d$ transition-metal surfaces, $\operatorname{Rh}(111)$ and $\operatorname{Pd}(111)$, the interactions are strong with small $d_{\mathrm{eq}}$ as in the case of the nanotube adsorptions. In more detail, the graphene on $\operatorname{Rh}(111)$ and $\operatorname{Pd}(111)$ is corrugated with varying $d_{\text {eq }}$. For
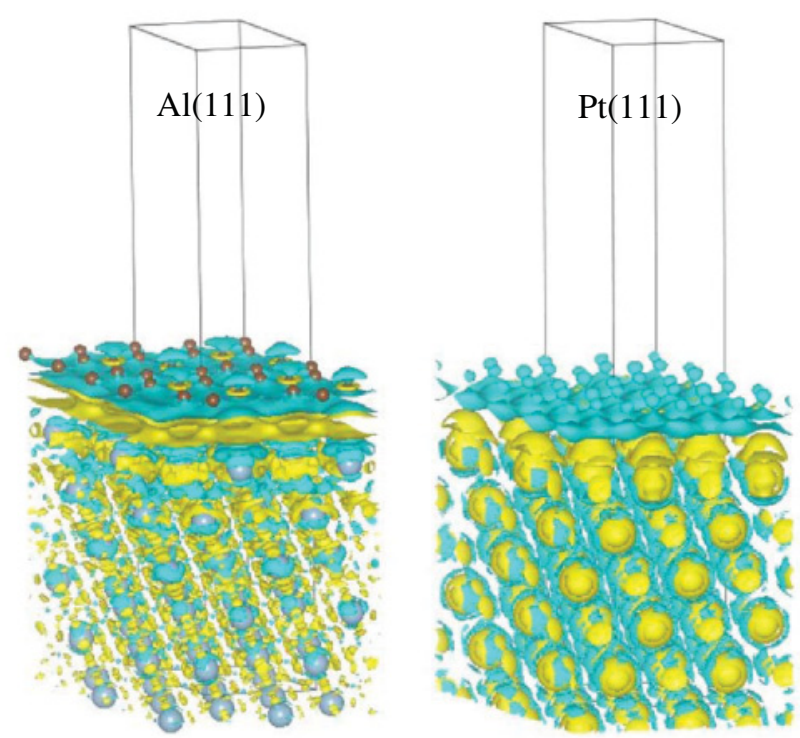

FIG. 7. (Color online) Difference charge density, $\Delta n(\mathbf{r})$, defined by Eq. (10), for the systems of graphene/Al(111) and graphene/Pt(111). The blue (darker shaded) and yellow (lighter shaded) domains show the electron depleted $[\Delta n(\mathbf{r})<0]$ and electron accumulated $[\Delta n(\mathbf{r})>0]$ regions, respectively, as in Fig. 2.

graphene/Rh(111) we have $d_{\mathrm{eq}} \sim 2.13 \AA$ for a carbon atom on the top site (over the metal atom), $d_{\mathrm{eq}} \sim 2.38 \AA$ for a carbon atom on the fcc(111) hollow site, and other $d_{\mathrm{eq}}$ values between the two extremes for the remaining 16 atoms in the supercell with the graphene- $(3 \times 3) /(\sqrt{7} \times \sqrt{7}) R 19.1^{\circ}$ structure. This corrugation of $\sim 10 \%$ is also found for graphene/ $\operatorname{Pd}(111)$. These corrugations are specific to the supercell employed in the present calculations and different results may be obtained if we use a different supercell. We also note that this corrugation is not the same as the moiré structures, which have been observed in the case of graphene physisorption on metals. ${ }^{33,35,36}$ We note the difference between $4 d$ and $5 d$ transition-metal surfaces in the graphene adsorption. Wang and $\mathrm{Che}^{32}$ have made detailed analyses on the origins of distinctly different behaviors of Pd and Pt contacts on graphene, which should also be addressed to the difference between $\mathrm{Rh}$ and $\mathrm{Ir}$ in the graphene adsorptions. They have argued that the electron exchange transfer between the Pd and graphene, which is absent between the Pt and

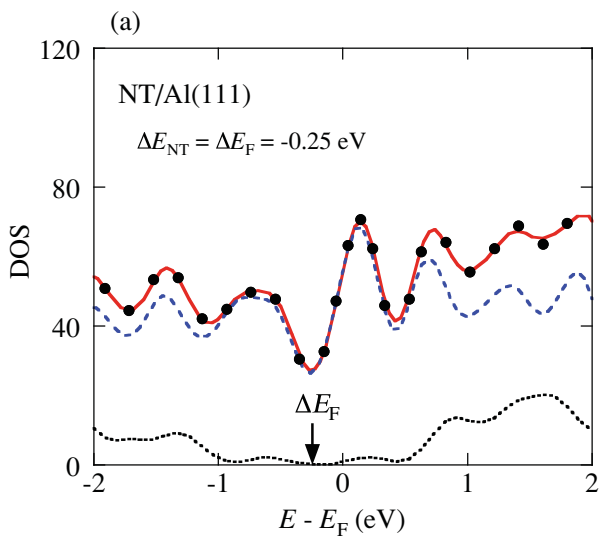

(b)

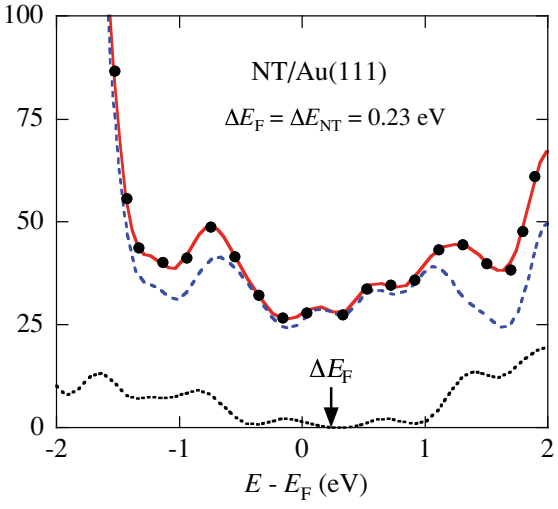

FIG. 6. (Color online) Density of states of the systems of an armchair $(10,10)$ nanotube (NT) on (a) $\mathrm{Al}(111)$ and (b) $\mathrm{Au}(111)$ surfaces. These results are counterparts of those in Fig. 4 and were obtained by less accurate electronicstructure calculations using $1 \times 5 \times 1$ kpoint MP mesh. 

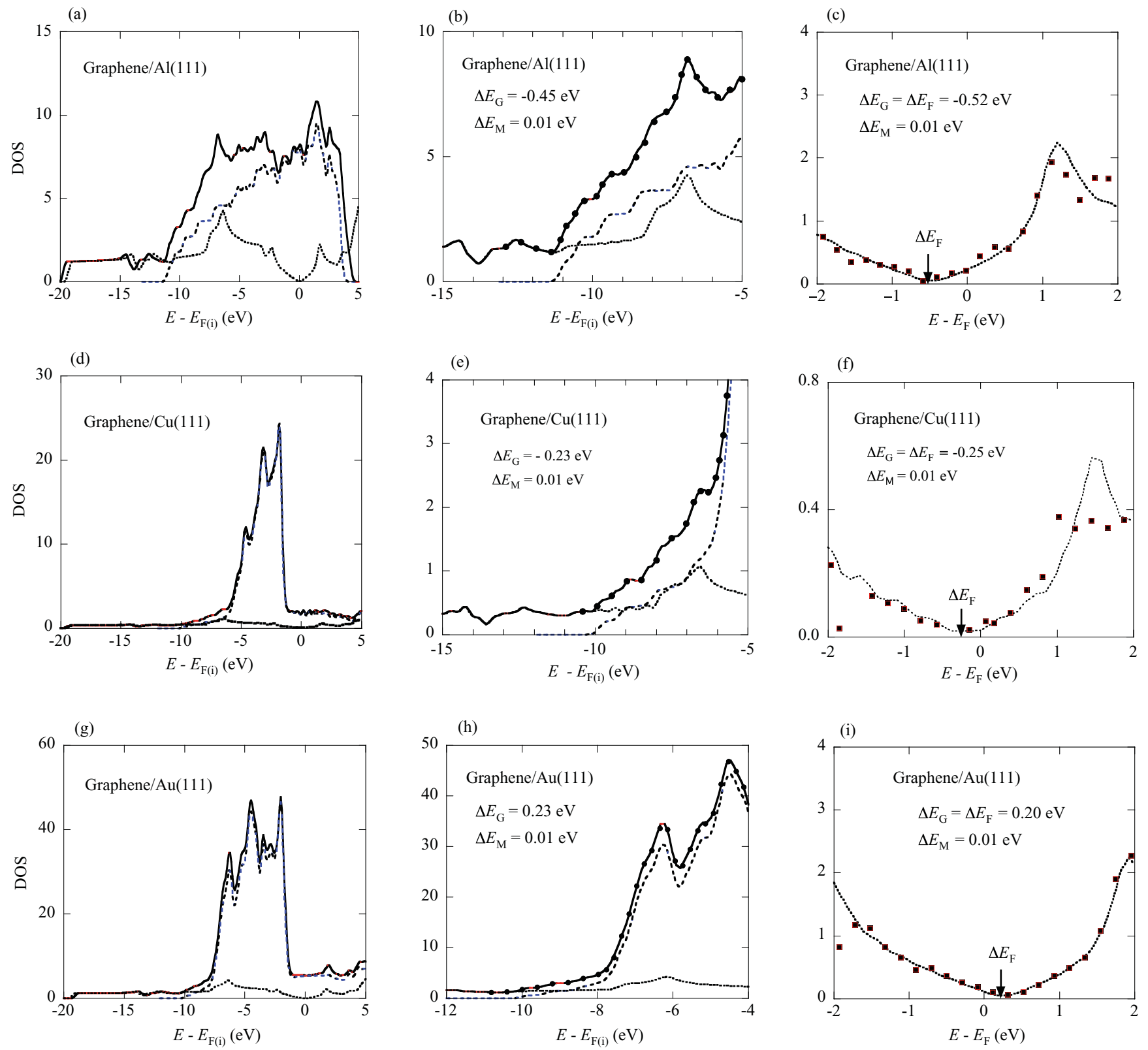

FIG. 8. (Color online) Density of states of the systems of graphene $(\mathrm{G})$ on $\mathrm{Al}, \mathrm{Cu}$, and $\mathrm{Au}(111)$ surfaces. The squares show $\rho_{\mathrm{NT}}^{\text {rigid }}(E)$ defined by Eq. (12). The meanings of other symbols and lines are similar to those in Fig. 3.

graphene, is responsible for this difference. Unfortunately, their argument cannot be traced in the present analyses based on the DFT calculations.

Figure 7 shows the difference charge densities, $\Delta n(\mathbf{r})$, for the systems of graphene $(\mathrm{G})$ on $\mathrm{Al}(111)$ and $\mathrm{Pt}(111)$ surfaces as typical examples of physisorption. Here, $\Delta n(\mathbf{r})$ is defined in a way similar to that in Eq. (10), in which nanotube (NT) is replaced by graphene $(\mathrm{G})$. For graphene/Al(111) the transferred electrons distribute in the interfacial region near the graphene, indicating that graphene is heavily $n$ doped as it is more clearly seen in terms of the Fermi-level shift, which we discuss later. On the other hand, for graphene/Pt(111) electrons are transferred from the whole region of graphene to the substrate, indicative of $p$-type doping of graphene, which is also consistent with the Fermi-level shift.
Figure 8 illustrates DOS for graphene/Al(111), graphene/Cu(111), and graphene/Au(111) as examples of physisorption. Here, as in the case of nanotube adsorption, $E_{\mathrm{F}(i)}$ is the Fermi energy of each system: $i=\mathrm{G} / \mathrm{M}$ (graphene/metal), $\mathrm{G}$ (isolated graphene), and $\mathrm{M}$ (isolated metal substrate); we use $E_{\mathrm{F}} \equiv E_{\mathrm{F}(\mathrm{G} / \mathrm{M})}$ as before. The results in Figs. 8(b), 8(e), and 8(h) indicate that the lower-energy bands show uniform shifts by $\Delta E_{\mathrm{G}}=-0.45$, -0.23 , and $0.23 \mathrm{eV}$ on $\mathrm{Al}, \mathrm{Cu}$, and $\mathrm{Au}(111)$ surfaces, respectively. Finite energy-band shifts of the substrate, $\Delta E_{\mathrm{M}} \approx 0.01 \mathrm{eV}$, are again an artifact due to finite-size effect. The rigid-band picture is not strictly valid in the sense that $\pi$ bands in the vicinity of the Dirac point show extra downward shifts. In the above cases, the total shifts of graphene $\pi$ bands are given by lower values of $\Delta E_{\mathrm{G}}=-0.52,-0.25 \mathrm{eV}$, and 
$0.20 \mathrm{eV}$, respectively, which are nothing but the Fermi-level shift, $\Delta E_{\mathrm{F}}$, of graphene. These values were obtained from the band-structure calculations (not shown here) because the graphene DOS, $\rho_{\mathrm{G}}(E)$, is small and structureless in the relevant range, which makes it difficult to accurately estimate $\Delta E_{\mathrm{F}}$ by rigid-band fitting. Here we use a quantity defined by

$$
\rho_{\mathrm{G}}^{\mathrm{rigid}}(E)=\rho_{\mathrm{G} / \mathrm{M}}(E)-\rho_{\mathrm{M}}\left(E-\Delta E_{\mathrm{M}}\right),
$$

which is the graphene DOS in the rigid-band model. The results for $\rho_{\mathrm{G}}^{\text {rigid }}(E)$ are, in fact, in good agreement with $\rho_{\mathrm{G}}(E-$ $\left.-\Delta E_{\mathrm{F}}\right)$ using the above lower values of $\Delta E_{\mathrm{F}}$ [Figs. 8(c), $8(\mathrm{f})$, and $8(\mathrm{i})]$. The above differences in the energy shifts indicate that the $\pi$ band downward shifts with respect to lower-energy bands are given by $0.07,0.02$, and $0.03 \mathrm{eV}$ for graphene on $\mathrm{Al}, \mathrm{Cu}$, and $\mathrm{Au}(111)$ surfaces, respectively. The extra $\pi$-orbital downward shifts for $\mathrm{Ag}$, Ir, and $\mathrm{Pt}(111)$ surfaces are $0.05,0.0$, and $0.05 \mathrm{eV}$, respectively. These $\pi$-orbital shifts may be interpreted as an effect of hybridization between $\pi$ orbitals and metal valence states as suggested by Grüneis and Vyalikh $^{38}$ for graphene/Ni(111), in which $\pi$ orbitals hybridize with the Ni $3 d$ bands. We find that the present results for the Fermi-level shift are in good agreement with similar calculations by Khomyakov et al. ${ }^{25}$ Recent STS studies ${ }^{34}$ of local electron DOS of graphene on metallic $\mathrm{Au} / \mathrm{Cr} / \mathrm{SiO}_{2} / \mathrm{Si}$ substrate show that substrate causes $p$-type doping of graphene with $\Delta E_{\mathrm{F}}=0.35-0.40 \mathrm{eV}$, which is in accordance with the DFT calculations for graphene/Au(111). The present results for graphene/Pt(111), $d_{\mathrm{eq}}=3.21 \AA$ and $\Delta E_{\mathrm{F}}=0.29 \mathrm{eV}$, are in very good agreement with the experiments, ${ }^{31} d_{\mathrm{eq}}=3.30 \pm$ $0.05 \AA$ and $\Delta E_{\mathrm{F}}=0.30 \pm 0.15 \mathrm{eV}$. However, this almost perfect agreement might be fortuitous in view of the limited accuracy of the DFT approximations. The present result for graphene/ $\operatorname{Ir}(111)$ indicates that graphene is slightly $p$ doped with $\Delta E_{\mathrm{F}}=0.05 \mathrm{eV}$, which is also in good agreement with the experimental result, ${ }^{39} \Delta E_{\mathrm{F}}=0.10 \pm 0.02 \mathrm{eV}$. Less accurate calculations using a supercell with graphene- $(2 \times 2) /(\sqrt{3} \times$ $\sqrt{3}) R 30^{\circ}$ structure predict that graphene on a $\operatorname{Ir}(111)$ surface is slightly $n$ doped with $\Delta E_{\mathrm{F}}=-0.05$, indicating that the absorption details in this case are to some extent influenced by the strain of metal substrates.

In Fig. 5, the results of DFT calculations for the Fermi-level shift as functions of the WF difference, $\Delta W=W_{\mathrm{M}}-W_{\mathrm{G}}$, are compared with the phenomenological model given by Eq. (9). The DFT results are well on a single line of the model with $\Delta V_{\mathrm{c}}=0.88 \mathrm{eV}$ except for $\mathrm{Au}(111)$ and $\operatorname{Ir}(111)$ surfaces. This value of $\Delta V_{\mathrm{c}}$ is in good agreement with the explicit evaluations of Khomyakov et al., ${ }^{25}$ who have obtained $\Delta V_{\mathrm{c}} \approx 0.90 \mathrm{eV}$ for graphene $/ \mathrm{Cu}(111)$ and used this value for other metal surfaces. The present results imply that the value of $\Delta V_{\mathrm{c}}$ is different in some cases, e.g., $\Delta V_{\mathrm{c}} \approx 0.70$ and $1.05 \mathrm{eV}$ for $\mathrm{Au}(111)$ and $\operatorname{Ir}(111)$ surfaces, respectively, where we used the experimental value of $\Delta E_{\mathrm{F}}=0.10 \mathrm{eV}$ for the latter case in estimating $\Delta V_{\mathrm{c}}$ through Eq. (9).

Finally, we would like to comment on the accuracy of the DFT calculations in the local (LDA) or semilocal (GGA) approximation. These approximations are probably insufficient for the systems of a nanotube or graphene on metal surfaces and it is likely that van der Waals (vdW) interactions must be taken into account for obtaining more accurate descriptions, as suggested by Fuentes-Cabrera et al. ${ }^{37}$ in their studies on the graphene/Ni(111) system. The adsorption geometries and nature of bonding at the graphene/metal interfaces have actually been investigated using the vdW density functionals (vdW-DFs). ${ }^{27,28}$ The equilibrium distances, $d_{\mathrm{eq}}$, obtained by one of such calculations, are $\sim 3.50 \AA$, indicative of physisorption, even for $\mathrm{Co}, \mathrm{Ni}$, and $\mathrm{Pd}(111)$ surfaces,${ }^{27}$ which are different from the LDA results and in contradiction to the experiments. Hamada and Otani ${ }^{28}$ have used varying types of $\mathrm{vdW}-\mathrm{DF}$ and found that the above interfacial properties depend on the type of vdW-DF and the best one yields the results for $d_{\text {eq }}$ in good agreement with available experiments and the LDA results. These results suggest that the LDA yields reasonable results for the interfacial geometries of the graphene/metal systems and is even better than some vdW-DFs with uncertain accuracy. However, we should note that the adsorption energies predicted with the LDA are too small, by a factor of $\sim 2$ in the case of physisorption, compared with the vdW-DF results and, probably, with experiments. The interfacial properties other than the adsorption energy may be well predicted by the DFT (LDA) calculations. These natures of vdW interactions and the LDA have actually been confirmed for the energetics of interlayer binding of graphite. ${ }^{51}$

\section{CONCLUSIONS}

In conclusion, we have elucidated adsorption characteristics of a metallic SWNT and graphene on a series of metal surfaces with particular emphasis on the transfer doping. The interactions between the nanotube and the surfaces of $\mathrm{Al}$ and noble metals $(\mathrm{Ag}, \mathrm{Cu}$, and $\mathrm{Au})$ are found to be weak and typical of physisorption, enabling one to define the Fermi-level shift, $\Delta E_{\mathrm{F}}$, characterizing type and degree of transfer doping. The present DFT result of $\Delta E_{\mathrm{F}}$ is in good agreement with the previous estimations and experiments for NT/Au(111). On the other hand, the nanotube adsorptions on the transition-metal surfaces (Rh, Pd, Ir, and Pt) are typical of chemisorption with small separations and large binding between the nanotube and substrates. Transfer doping of graphene on metal surfaces is more substantial than that for nanotube/metals because of more efficient, flat interfaces between graphene and substrates. Our reexaminations revealed that graphene is physisorbed not only on $\mathrm{Al}$ and nobles metal surfaces but also on the $5 d$ transitionmetal surfaces, $\operatorname{Ir}(111)$ and $\operatorname{Pt}(111)$, and the Fermi-level shifts of graphene on $\operatorname{Ir}(111)$ and $\operatorname{Pt}(111)$ are in good agreement with available experiments. The different adsorption behaviors of a nanotube and graphene on $5 d$ transition-metal surfaces may be explained by the curvature-enhanced hybridization of $\pi$ orbitals with the valence states of substrate, which is absent in the case of graphene. We found that the cause of the Fermi-level shift of graphene is twofold in the case of physisorption: One is a usual band shift due to the potential difference and the other is the extra downward shift of $\pi$ orbitals, which is also due to the hybridization with the valence states of substrate. The interactions between graphene and $4 d$ transition-metal surfaces, $\operatorname{Rh}(111)$ and $\operatorname{Pd}(111)$, are again strong enough to form bonding as in the case of the nanotube on these metal surfaces. This difference between graphene adsorptions on $4 d$ and $5 d$ transition-metal surfaces must be 
explained by a sophisticated mechanism, such as an exchange transfer. ${ }^{32}$

We have also developed a phenomenological model, which provides a useful insight into transfer doping through a relation between the Fermi-level shift and the WF difference and is applicable to both nanotube and graphene on metal surfaces. We used this model together with the DFT calculations and estimated quantum-mechanical effect, which arises from the overlap of adsorbate and substrate wave functions and is substantiated as the potential step in the adsorbate with respect to the substrate. This effect is found to be somewhat at variance in the graphene adsorptions, suggesting that effect is viable to change depending on the electronic structure of substrates. On the other hand, this effect is fairly uniform and independent of substrates in the case of nanotube physisorption on metals.

\section{ACKNOWLEDGMENTS}

This work was support by a Grant-in-Aid for Scientific Research from the JSPS (No. 20540394). The computations in this work were performed using the facilities of the Information Processing Center, Iwate University, and the Supercomputer Center at the ISSP, University of Tokyo.
*Corresponding author: hasegawa@iwate-u.ac.jp

${ }^{1}$ S. Iijima, Nature (London) 354, 56 (1991).

${ }^{2}$ K. S. Novoselov, A. K. Geim, S. V. Morozov, D. Jiang, Y. Zhang, S. V. Dobonos, I. V. Grigorieva, and A. A. Firsov, Science 306, 666 (2004).

${ }^{3}$ R. Saito, G. Dresselhaus, and M. S. Dresselhaus, Physical Properties of Carbon Nanotubes (Imperial College Press, London, 1998).

${ }^{4}$ J.-C. Charlier, X. Blase, and S. Roche, Rev. Mod. Phys. 79, 677 (2007).

${ }^{5}$ Y. H. Wu, T. Yu, and Z. X. Shen, J. Appl. Phys. 108, 071301 (2010).

${ }^{6}$ M. P. Anantram and F. Léonard, Rep. Prog. Phys. 69, 507 (2006).

${ }^{7}$ P. Avouris, Nano Lett. 10, 4285 (2010).

${ }^{8}$ F. Banhart, Nanoscale 1, 201 (2009).

${ }^{9}$ J. W. G. Wildöer, L.C. Venema, A. G. Rinzler, R. E. Smalley, and C. Dekker, Nature (London) 391, 59 (1998).

${ }^{10}$ T. W. Odom, J.-L. Huang, P. Kim, and C. M. Lieber, Nature (London) 391, 62 (1998).

${ }^{11}$ A. Rubio, D. Sanchez-Portal, E. Artacho, P. Ordejon, and J. M. Soler, Phys. Rev. Lett. 82, 3520 (1999).

${ }^{12}$ Y. Xue and S. Datta, Phys. Rev. Lett. 83, 4844 (1999).

${ }^{13}$ L. C. Venema, J. W. Janssen, M. R. Buitelaar, J. W. G. Wildöer, S. G. Lemay, L. P. Kouwenhoven, and C. Dekker, Phys. Rev. B 62, 5238 (2000).

${ }^{14}$ S. G. Lemay, J. W. Jassen, M. van den Hout, M. Mooij, M. J. Bronikowski, P. A. Willis, R. E. Smalley, L. P. Kouwenhoven, and C. Dekker, Nature (London) 412, 617 (2001).

${ }^{15}$ M. Ouyang, J.-L. Huang, C. L. Cheung, and C. M. Lieber, Science 292, 702 (2001)

${ }^{16}$ A. Maiti and A. Ricca, Chem. Phys. Lett. 395, 7 (2004).

${ }^{17}$ B. Shan and K. Cho, Phys. Rev. B 70, 233405 (2004).

${ }^{18}$ S. Okada and A. Oshiyama, Phys. Rev. Lett. 95, 206804 (2005).

${ }^{19}$ C. E. Giusca, Y. Tison, and S. R. P. Silva, Phys. Rev. B 76, 035429 (2007).

${ }^{20}$ H.-J. Shin, S. Clair, Y. Kim, and M. Kawai, Appl. Phys. Lett. 93, 233104 (2008).

${ }^{21}$ K. Nishidate and M. Hasegawa, Phys. Rev. B 81, 125414 (2010).

${ }^{22}$ C. Oshima and A. Nagashima, J. Phys. Condens. Matter 9, 1 (1997).

${ }^{23}$ J. Wintterlin and M.-L. Bocquet, Surf. Sci. 603, 1841 (2009).

${ }^{24}$ G. Giovannetti, P. A. Khomyakov, G. Brocks, V. M. Karpan, J. van den Brink, and P. J. Kelly, Phys. Rev. Lett. 101, 026803 (2008).

${ }^{25}$ P. A. Khomyakov, G. Giovannetti, P. C. Rusu, G. Brocks, J. van den Brink, and P. J. Kelly, Phys. Rev. B 79, 195425 (2009).
${ }^{26}$ Q. Ran, M. Gao, X. Guan, Y. Wang, and Z. Yu, Appl. Phys. Lett. 94, 103511 (2009).

${ }^{27}$ M. Vanin, J. J. Mortensen, A. K. Kelkkanen, J. M. Garcia-Lastra, K. S. Thygesen, and K. W. Jacobsen, Phys. Rev. B 81, 081408(R) (2010).

${ }^{28}$ I. Hamada and M. Otani, Phys. Rev. B 82, 153412 (2010).

${ }^{29} \mathrm{Z}$. Xu and M. J. Buehler, J. Phys. Condens. Matter 22, 485301 (2010).

${ }^{30}$ A. B. Preobrajenski, M. L. Ng, A. S. Vinogradov, and N. Martensson, Phys. Rev. B 78, 073401 (2008).

${ }^{31}$ P. Sutter, J. T. Sadowski, and E. Sutter, Phys. Rev. B 80, 245411 (2009).

${ }^{32}$ Q. J. Wang and J. G. Che, Phys. Rev. Lett. 103, 066802 (2009).

${ }^{33}$ Z. Klusek, P. Dabrowski, P. Kowalczyk, W. Koziowski, W. Olejniczak, P. Blake, M. Szybowicz, and T. Runka, Appl. Phys. Lett. 95, 113114 (2009)

${ }^{34}$ B. Wang, M. Caffio, C. Bromley, H. Früchtl, and R. Schaub, ACS Nano 4, 5773 (2010).

${ }^{35}$ M. Gao, Y. Pan, C. Zhang, H. Hu, R. Yang, H. Lu, J. Cai, S. Du, F. Liu, and H.-J. Gao, Appl. Phys. Lett. 96, 053109 (2010)

${ }^{36}$ L. Gao, J. R. Guest, and N. P. Guisinger, Nano Lett. 10, 3512 (2010).

${ }^{37}$ M. Fuentes-Cabrera, M. I. Baskes, A. V. Melechko, and M. L. Simpson, Phys. Rev. B 77, 035405 (2008).

${ }^{38}$ A. Grüneis and D. V. Vyalikh, Phys. Rev. B 77, 193401 (2008).

${ }^{39}$ I. Pletikosic, M. Kralj, P. Pervan, R. Brako, J. Coraux, A. T. N'Diaye, C. Busse, and T. Michely, Phys. Rev. Lett. 102, 056808 (2009).

${ }^{40}$ G. Kresse and J. Furthmüller, Phys. Rev. B 54, 11169 (1996); Comput. Mater. Sci. 6, 15 (1996).

${ }^{41}$ D. M. Ceperley and B. J. Alder, Phys. Rev. Lett. 45, 566 (1980).

${ }^{42}$ J. P. Perdew and A. Zunger, Phys. Rev. B 23, 5048 (1981).

${ }^{43}$ G. Kresse and D. Joubert, Phys. Rev. B 59, 1758 (1999).

${ }^{44}$ H. J. Monkhorst and J. D. Pack, Phys. Rev. B 13, 5188 (1976).

${ }^{45}$ O. Gülseren, T. Yildirim, S. Ciraci, and C. Kilic, Phys. Rev. B 65, 155410 (2002).

${ }^{46}$ K. Nishidate and M. Hasegawa, Phys. Rev. B 78, 195403 (2008).

${ }^{47}$ B. Shan and K. Cho, Phys. Rev. Lett. 94, 236602 (2005).

${ }^{48}$ V. Barone, J. E. Peralta, J. Uddi, and G. E. Scuseria, J. Chem. Phys. 124, 024709 (2006).

${ }^{49}$ W. S. Su, T. C. Leung, and C. T. Chan, Phys. Rev. B 76, 235413 (2007).

${ }^{50}$ CRC Handbook on Chemistry and Physics (CRC, Boca Raton, FL, 2004), pp. 12-124.

${ }^{51}$ M. Hasegawa and K. Nishidate, Phys. Rev. B 70, 205431 (2004). 